\title{
Origin of the additional chromosome in Down's syndrome: a study of 20 families
}

\author{
D F ROBERTS AND M H CALLOW \\ Department of Human Genetics, University of Newcastle upon Tyne, 19 Claremont Place, \\ Newcastle upon Tyne NE2 4AA
}

SUMMARY In 20 families with a Down's syndrome child, heteromorphism of chromosome 21 was sought in the parents and proband. Information on the meiotic division and the parent of origin was given in 16 families, the majority of errors being maternal in origin, predominantly at meiosis I, though the paternal contribution is also appreciable. Probabilities of parental and meiotic division origin are calculated.

The first published studies tracing the origin of the extra chromosome 21 in Down's syndrome were those of de Grouchy ${ }^{1}$ and Juberg and Jones, ${ }^{2}$ who in two families where the rare $21 \mathrm{p}-$ Christchurch chromosome was present were able to show that non-disjunction had taken place at the second meiotic division in oogenesis. Subsequently, by quinacrine fluorescence techniques, the No 21 chromosomes were found to be polymorphic, showing inherited variants ${ }^{3}{ }^{4}$ which had no abnormal effect on cell division. These techniques therefore allow the source of the extra chromosome in trisomic conditions to be traced in those matings where the chromosomes of the parents are sufficiently heterogeneous. With the refinement of technique by Overton et al, ${ }^{5}$ in which the photographic exposure for each chromosome is standardised by the use of a fluorescent illuminator and automatic exposure control, and the entire chromosome is examined in sequential prints, more heteromorphism of slighter degree can be distinguished. This technique was applied in the present study.

\section{Material and methods}

Twenty families with a Down's syndrome child were examined and blood specimens were obtained from the 20 patients, their 20 mothers, and 20 fathers. These families were a random selection from those referred for cytogenetic diagnosis or counselling during the period of the study. Permission was obtained from the family doctor to visit the family and collect the specimens. From each subject $10 \mathrm{ml}$ venous blood was taken into a heparinised bottle. Received for publication 29 December 1979
As controls for the laboratory procedures, specimens were obtained from normal subjects with no family history of Down's syndrome, consisting of 21 members of staff from this Department and 39 other normal subjects.

The specimens were cultured by the procedure standard in this Department. Ten drops of whole blood were cultured in $5 \mathrm{ml}$ media with filtered fetal calf serum. Phytohaemagglutinin was used as the mitogen. The cultures were incubated at $37^{\circ} \mathrm{C}$ for 70 hours and before fixation were treated with colcemid for 90 minutes, followed by $0.075 \mathrm{~mol} / \mathrm{l}$ potassium chloride solution as hypotonic for 20 minutes. The cell suspension was fixed in $3: 1$ methanol:acetic acid mixture, with a minimum of four changes of fixative. Centrifugation was at $\mathbf{2 0 0 0}$ rpm for five minutes. After overnight refrigeration, fresh fixative was again added and the cell suspension was dropped on to clean slides which had been kept in ethyl alcohol and wiped with a tissue before use. Slides were air or flame dried and labelled.

Slides were stained for a minimum of 15 minutes in $0.5 \%$ quinacrine dihydrochloride in methanol, washed, and mounted in distilled water. The cover slips were sealed to prevent evaporation. The slides were examined under an HBO 200 mercury vapour incident light source, FITC5 excitation filters, and a barrier filter OG515. Photography was by Kodak Tri-X Pan $35 \mathrm{~mm}$ film, with exposure times of 15 to 25 seconds. To maintain constant intensity of light, exposure time was increased as the lamp aged, and a new lamp was fitted every 180 hours. At least ten metaphase spreads were photographed per subject. Five to ten of the best quality negatives were printed, using sequential printing according to the method 
of Overton et al..$^{5}$ A piece of card with a frame cut in it was moved along a strip of photographic paper for serial exposures of each chromosome 21. The timing of exposures differed according to the intensity of the negative, but each chromosome was given a range of exposure from under- to overexposed, usually at one second increments (fig 1). This enabled all the features and the banding patterns of each chromosome to be fully analysed.

Since the variant regions of the chromosomes cover a wide range of intensity and size and apparently vary continuously, five levels of intensity of fluorescence are recognised (Paris Conference, 1971). In the present study scoring was either positive (the brilliant and intense levels) or normal (the three lower intensity levels). The variants of the chromosomes 21 were scored in detail. The short arms, stalk, and satellites vary in size, and these were each scored on a four point scale (absent, small, medium, and large). The fluorescent intensity of the short arm and satellites were also recorded.

Using this procedure it is possible in the majority of cases to distinguish between the parental chromosomes. In a given mating the chromosomes are coded abcd if four can be distinguished and abcc or acbc if three (if two are morphologically identical), and from the code the origin of the additional chromosome in the trisomic child can be inferred. In the mating $a b$ male by cd female, if the offspring has chromosomes abc, then non-disjunction must have occurred at the first meiotic division in the father.
If the offspring has chromosomes acc, then nor disjunction occurred at the second meiotic division. in the mother. Trisomy arising from post-zygotic non-disjunction with subsequent loss of the mone somic cell line cannot be distinguished from that arising in the second meiotic division.

In practice, such an ideal mating in which fou chromosomes can each be identified rarely occurs However, a limited amount of information can be drawn from all matings. For example, in the mating ab male by cc female, a trisomy acc could be produced by non-disjunction at the first or secone meiotic division in the mother, but not by nor disjunction at either division in the father. However all unions contribute information on the probs ability of non-disjunction at a particular meiote division in a given parent, and only in the case where all four parental chromosomes are identical are thess probabilities equal. Hence the cases in a series can be combined, provided that they are not selected, t $\overrightarrow{8}$ give estimates of the probability of non-disjunction occurring in each meiotic division in each parent $\frac{9}{8}$ If $p$ is the probability of paternal origin given non disjunction, $1-p$ is the probability of maternal origir $\overrightarrow{0}$ If $f$ is the probability of first division non-disjunctio given maternal origin, $1-f$ is the probability of second division non-disjunction in the female. If $f+d$ is the probability of a first division error given paterna origin, then 1-f-d is the probability of secon division non-disjunction in the male. From these the marginal probabilities of the four mutually

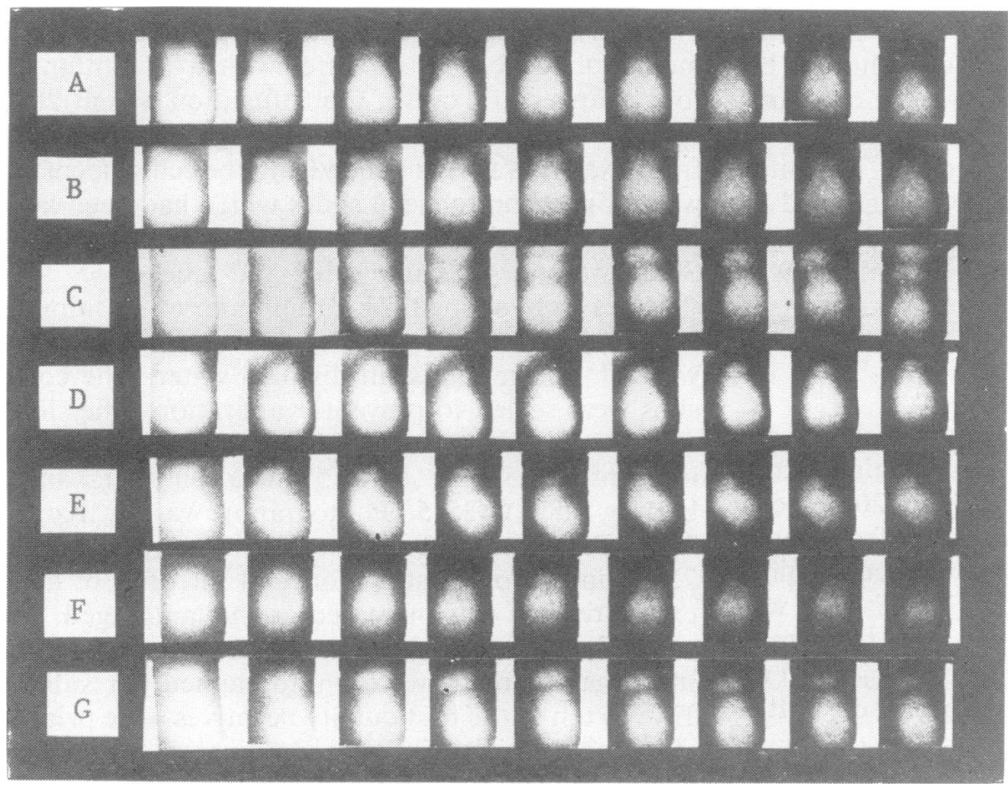

FIG 1 Seven different chromosomes 21 ( $A$ to $G)$ to illustrate the methodo 
exclusive types of non-disjunction can be calculated by straightforward maximum likelihood methods.

\section{Results}

The results of the investigation are set out in table 1 . All families are given, whether interpretable simply or not, in order to avoid the bias that partial reporting of the data would introduce.

In nine cases the error could be definitely ascribed to one parent or another. Of these the mother was implicated in eight (for example, fig 2) and the father in one (fig 3). The case where the male parent was definitiely involved indicated error at the first meiotic division. Where the female was the source, in four the first meiotic division was indicated, in one the second division was indicated, and in three an error at either could have been responsible. In four cases all four parental chromosomes were morphologically identical. In the remainder, although the supernumerary chromosome might have been attributed to either parent, one or more of the four possible origins could be eliminated, and so some indication of origin was given. Error at the

TABLE 1 Observed mating types producing a Down's syndrome child, parental ages at time of birth, and conditional probabilities of sources of non-disjunction

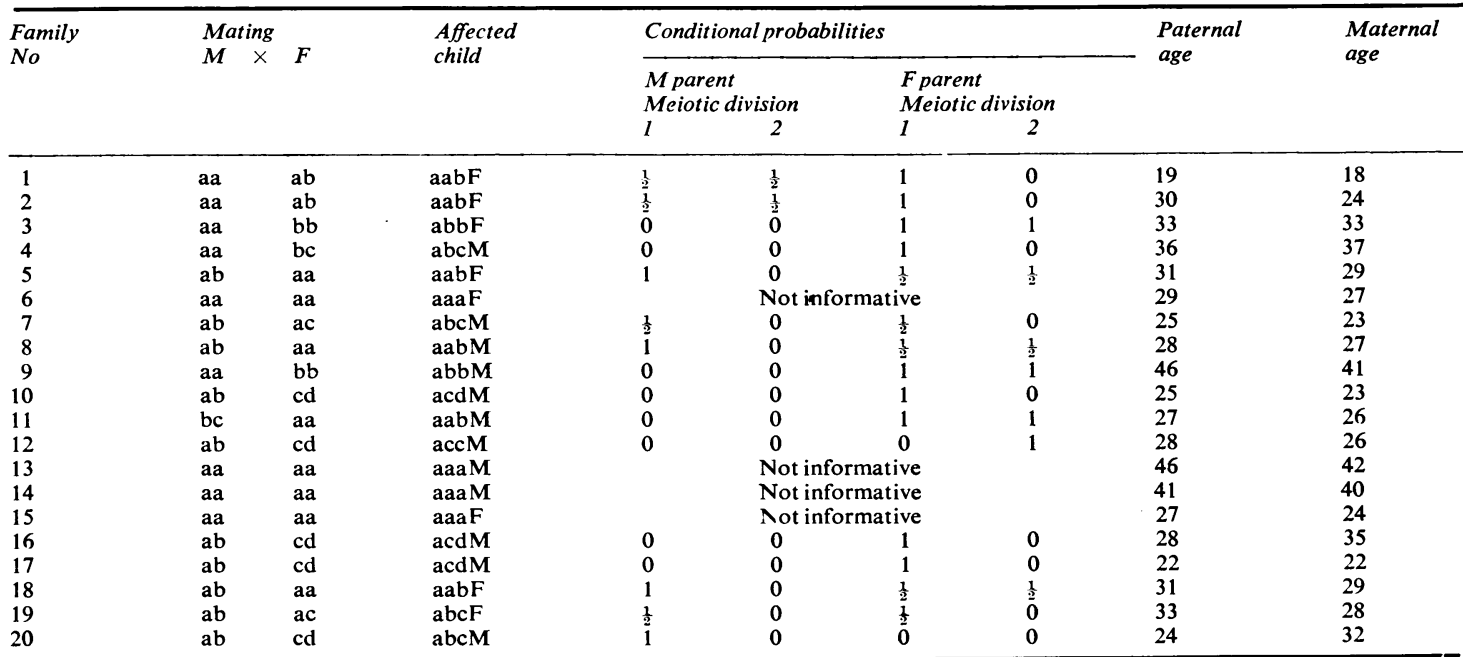

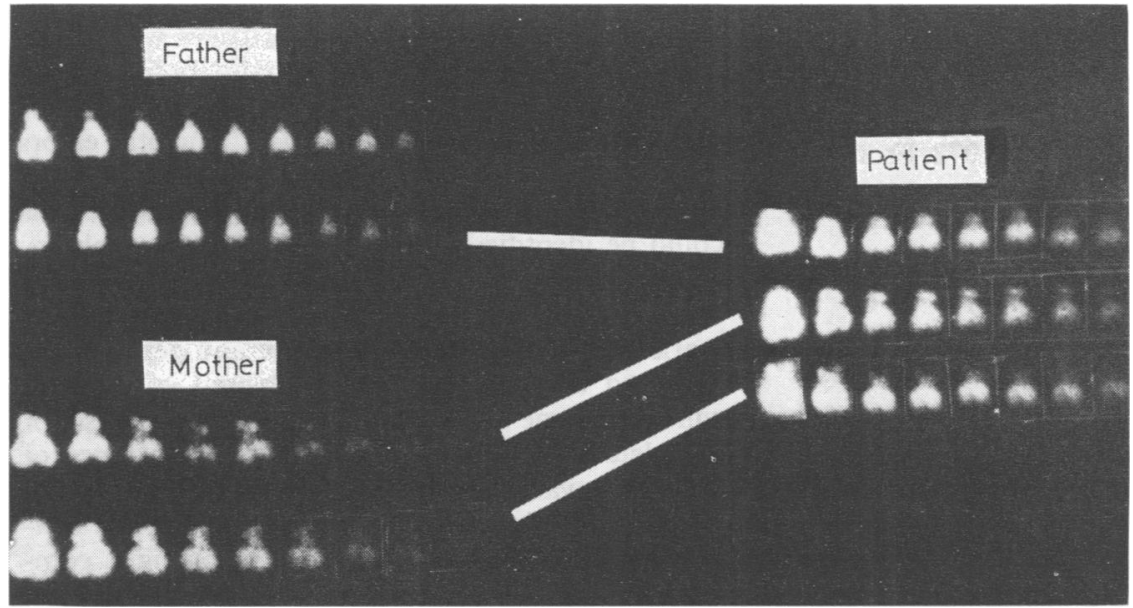

FIG 2 A maternal first meiotic division is the source of the error. 


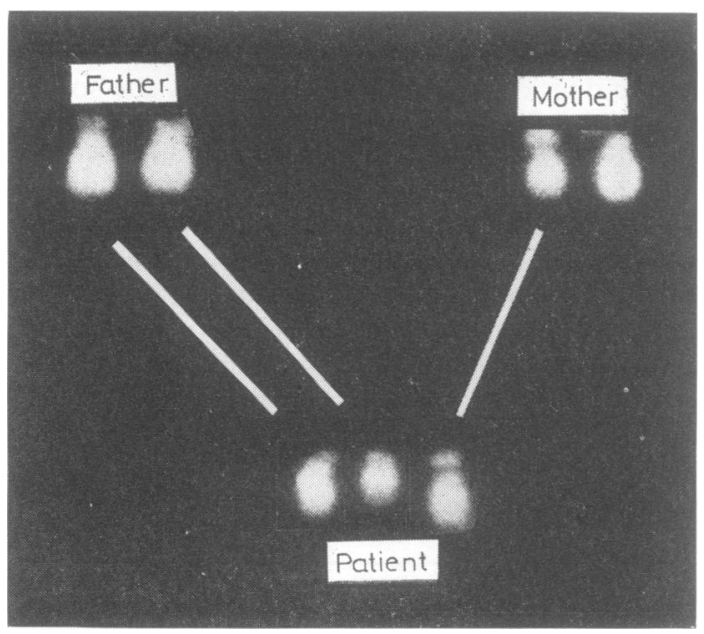

FIG 3 Error at the paternal first meiotic division.

The second maternal chromosome is excluded by

its greater fluorescent intensity as well as its morphology.

first meiotic division in either parent was indicated in one case. In two cases the first meiotic division in the mother, or either in the father, was suggested. In three cases the derivation appeared to be from either the first meiotic division in the father or either division in the mother. Combined with the information on the definitive assignations, of the 16 unions each with four possible sources of error, there are 33 that can be definitely eliminated. Of these, 14 are the second paternal meiotic division, nine the second maternal, eight the first paternal, and two the first maternal meiotic division.

These results are summarised in table 1 , together with the conditional probabilities for each case that the error in the child derived from non-disjunction in a given parent or division. From these the maximum likelihood estimates of the defined probabilities are calculated as: $p=0.136 \pm 0.093$, $\mathrm{d}=0.119 \pm 0.072, \mathrm{f}=\mathbf{0 . 8 8 1} \pm \mathbf{0} \cdot 072$. From these the probability at each of the four sources is as follows.

Male first division

Male second division

$\begin{array}{ll}p(f+d)) & 0 \cdot 136 \\ p(1-f-d) & 0 \cdot 0 \\ f(1-p) & 0 \cdot 761 \\ (1-p)(1-f) & 0 \cdot 103 .\end{array}$

Female first division

Female second division

$(1-p)(1-f)$

$0 \cdot 103$.

It is interesting that $d$, the measure of sex variation in the probability of non-disjunction at a given division, is not significantly different from 0 .

The parental ages are shown for the informative matings. Maternal errors definitely at first meiosis occurred at maternal ages of $22,23,35$, and 37 . The error at the maternal second meiosis occurred at a maternal age of 26. The paternal age in the firs meiosis error was 24 . The definite maternal errors either first or second meiosis occurred at maternaf ages 33, 41, and 26. From the small number of specimens examined, little conclusive can be ex pected, but the results indicate that maternal meiosis errors occur at all ages and that there is no tendenc多 for either late or early parental age to be associategs with a specific error.

\section{Discussion}

Reports of similar studies summarised in table show that, as in the present series, the majority of errors are maternal in origin, predominantly at meiosis I. Studies which have given parental age show that maternal meiosis I errors predominate 2 all ages. ${ }^{7}$ In the present series the ages of the parento responsible are distributed across the whole rang $\underline{\overrightarrow{\mathrm{e}}}$ from 18 to 41 .

However, comparison of published series is nof straightforward on account of the bias towards the detection of non-disjunction of events in the secon\& meiotic division, as noted by Robinson. ${ }^{8}$ There are seven possible mating types:

$$
\begin{aligned}
& a a \times b b \\
& a a \times a b \\
& a a \times b c \\
& a b \times a c \\
& a b \times c d \\
& a a \times a a \\
& a b \times a b .
\end{aligned}
$$

The information they give depends upon the karyo type of the offspring, and does not always allow. definite assignment of the error to a given meiotic division or parent. Among studies up to 1976 which reported mating types, all of the second meiotie division errors were found from matings where only this event could be detected. Initially, because there were few recognisable variant types of chromosome 21 , matings in which only second meiotic errors could be detected were more frequent than those in which both first and second meiotic non-disjunctions. could be identified. However, the improved photon graphic technique of Overton et $a l^{5}$ has shown an increased degree of heteromorphism "which wilb markedly increase the success rate and permit valid unbiased conclusions". ${ }^{7}$ But unbiased conclusions will only come from the reporting and analysis of complete series as here. Hence the importance of using all available information from all available? matings to calculate probabilities. There are rela- 0 tively few published series where reporting is complete; hence comparison is only made with the series of 266 cases of trisomy 21 for which the $\frac{\mathbb{Q}}{\Omega}$ 
TABLE 2 Definite assignments of non-disjunctional events in standard trisomy 21

\begin{tabular}{|c|c|c|c|c|c|c|c|}
\hline \multirow[t]{2}{*}{ Reference* } & \multicolumn{2}{|c|}{$\begin{array}{l}\text { lst meiotic } \\
\text { division }\end{array}$} & \multicolumn{2}{|c|}{$\begin{array}{l}\text { 2nd meiotic } \\
\text { division }\end{array}$} & \multicolumn{2}{|c|}{$\begin{array}{l}\text { Ist or } 2 \text { nd } \\
\text { meiotic division }\end{array}$} & \multirow{2}{*}{$\begin{array}{l}\text { No of } \\
\text { informative } \\
\text { cases }\end{array}$} \\
\hline & Mat & Pat & Mat & Pat & Mat & Pat & \\
\hline Bott et al (1975) & & & 3 & 4 & & & 7 \\
\hline de Grouchy (1970) & & & 1 & & & & 1 \\
\hline Dutrillaux (1975) & 1 & & 1 & & & & 2 \\
\hline Emberger and Taib (1975) & & & $i$ & & & & 1 \\
\hline Giraud et al (1975) & 1 & & 1 & & & & 2 \\
\hline Hansson and Mikkelsen (1978) & 9 & 3 & 6 & 4 & 4 & & 26 \\
\hline Hara and Sasaki (1975) & 1 & & 2 & 1 & 2 & & 6 \\
\hline Juberg and Jones (1970) & & & 1 & & & & 1 \\
\hline Kajii and Niikawa (1973) & 1 & & & & & & 1 \\
\hline Kajii et al (1976) & $3+$ & & & & & & 3 \\
\hline Licznerski and Lindsten (1972) & 1 & & & & & & 1 \\
\hline Magenis et al (1977) & 23 & 5 & 1 & 2 & & & 31 \\
\hline Moore et al (1976) & 1 & & & & & & 1 \\
\hline Mutton (1973) & & & 1 & & & & 1 \\
\hline Niikawa et al (1977)† & 3 & & 1 & & & & 4 \\
\hline Punnett et al (1973) & 1 & & & 1 & & & 2 \\
\hline Robinson (1973) & 4 & & & & & & 4 \\
\hline Sasaki and Hava (1973) & & & & 1 & & & 1 \\
\hline Schmidt et al (1976) & 22 & 1 & & & & & 23 \\
\hline Uchida (1973) & & & & 1 & & & 1 \\
\hline Wagenbichler et al (1976) & 7 & 4 & 2 & 3 & 1 & 1 & 18 \\
\hline Present study & 4 & 1 & 1 & & 3 & & 9 \\
\hline Total & 82 & 14 & 22 & 17 & 10 & 1 & 146 \\
\hline
\end{tabular}

* Full references available from the authors on request. $\dagger$ Spontaneous abortions.

matings are reconstructed by Hassold and Matsuyama. ${ }^{9}$ The present estimates of $p, d$, and $f$ $(0 \cdot 136,0 \cdot 119,0 \cdot 881)$ are not significantly different from their respective estimates of $0.241,0.003$, $0 \cdot 773$. Comparison was by the $\log$ ratio test giving a value of $\mathrm{G}=2 \cdot 94$, df $3, \mathrm{p}>0.05$.

While caution is necessary in drawing together information on the origin of the third chromosome in Down's syndrome, the evidence appears overwhelming that, despite the bias towards detection of errors that occurred at meiosis II, most non-disjunctions occur at meiosis I, and with this the probability estimates agree. The finding that non-disjunction from the female parent does not occur at meiosis II with the same frequency as meiosis $I$ is not surprising in view of the duration of the first meiotic stage in the female. First and second meiotic divisions may be influenced in quite different ways by quite different factors. The results so far also indicate that the male parent contributes the extra chromosome in an appreciable proportion of trisomy 21 cases, in the present study one out of 16 , and in published material 29 out of 127, informative cases, though the precise probability remains to be established. Finally, it is not yet clear whether there is sex variation in the probability of non-disjunction at a given meiotic division, and this is a key question that remains to be answered for the information it will give on the mechanisms responsible.

\section{References}

1 de Grouchy J. 21p-maternel en double exemplaire chez un trisomique 21. Ann Genet (Paris) 1970;13:52-5.

2 Juberg RC, Jones B. The Christchurch chromosome (Gp-) mongolism, erythroleukemia and an inherited $\mathrm{Gp}$ chromosome. N Engl J Med 1970;282:292-7.

${ }^{3}$ Casperssen T, Hulten M, Lindsten J, Zech L. Distinction between extra G-like chromosomes by quinacrine mustard fluorescence analysis. Exp Cell Res 1970;63:240-3.

4 Casperssen T, Lomakka G, Zech L. The 24 fluorescence patterns of the human metaphase chromosomes-distinguishing characters and variability. Hereditas 1971; 67: 89-102.

5 Overton KM, Magenis RE, Brady T, Chamberlin J, Parks M. Cytogenetic darkroom magic: now you see them, now you don't. Am J Hum Genet 1976;28:417-9.

6 Jacobs PA, Morton NE. Origin of human trisomics and polyploids. Hum Hered 1977;27:59-72.

7 Magenis RE, Overton KM, Chamberlin J, Brady T, Lovrien E. Parental origin of the extra chromosome in Down's syndrome. Hum Genet 1977;37:7-16.

8 Robinson $\mathbf{J}$. Origin of extra chromosome in trisomy 21. Lancet 1973 ;ii:131-3.

9 Hassold T, Matsuyama A. Origin of trisomies in human spontaneous abortions. Hum Genet 1979;46:285-94.

Requests for reprints to Professor D F Roberts, Department of Human Genetics, University of Newcastle upon Tyne, 19 Claremont Place, Newcastle upon Tyne NE2 4AA. 